\title{
James Sacré : une écriture de gestes de vie
}

\author{
Irati FERNÁNDEZ ERQUICIA \\ Universidad Nacional de Educación a Distancia (UNED) \\ fernandezirati@gmail.com
}

Recibido: $18 / 12 / 2015$

Aceptado: 08/04/2016

\begin{abstract}
Résumé
Dans la poésie française contemporaine, quelques poètes cherchent un retour et une remise en valeur de l'énonciation du sujet poétique. Au sein de ce panorama, le poète James Sacré constituerait un exemple de cette nouvelle approche qui voudrait retrouver l'expression du sujet poétique tout en évitant de tomber dans l'excès, l'ornementation et l'effusivité poétiques. Cette approche est mise en place à travers l'utilisation d'un langage simple, qui met en valeur la lisibilité, et qui n'hésite pas à prendre comme point de départ le côté le plus banal et anodin de la réalité. L'article étudie plus en profondeur l'un des procédés utilisés par le poète pour rétablir l'expression du sujet poétique, qui consiste en la mise en place d'une écriture de gestes de vie, ce qui montre le lien indéniable existant dans la poésie de Sacré entre la vie et les mots poétiques.
\end{abstract}

Mots clés : James Sacré, poésie française contemporaine, gestes, quotidien, mots, vie.

\section{James Sacré, una escritura de gestos de vida}

\begin{abstract}
Resumen
En la poesía francesa contemporánea, algunos poetas buscan un retorno y una revalorización de la enunciación del sujeto poético. Dentro de este panorama, el poeta James Sacré constituiría un ejemplo de este nuevo acercamiento que intenta reencontrar la expresión del sujeto poético, al mismo tiempo que intenta evitar caer en el exceso, la ornamentación y la efusividad poética. Este acercamiento se realiza a través de la utilización de un lenguaje simple, que valoriza la legibilidad, y que no duda en partir del aspecto más banal y anodino de la realidad. El articulo estudia con más atención uno de los procedimientos que el poeta utiliza para restablecer la expresión del sujeto poético, que consiste en recurrir a una escritura de gestos de vida, lo que muestra que existe una relación innegable en la poesía de Sacré entre la vida y la palabras poéticas.
\end{abstract}

Palabras clave: James Sacré, poesía francesa contemporánea, gestos, cotidianidad, palabras, vida.

\section{James Sacré, a writing of life gesture}

\begin{abstract}
In French contemporary poetry, some poets have wished to return to - and so to increase the value of - the enunciation of the poetic subject. In such poetic scenario, the poet James Sacré exemplifies a new approach that tries to re-establish contact with the expression of the poetic subject, albeit always avoiding the pitfalls of excessive ornamentation and poetic effusiveness. Based on the use of simple language, this approach attaches value to legibility and does not hesitate to tap into the most banal or dullest aspects of reality. This article studies one of the procedures used by the poet to reestablish the expression of the poetic subject. This procedure seeks to rewrite life gestures - a technique that evinces an unavoidable relationship between life and poetic words in the work of James Sacré.
\end{abstract}


Keywords: James Sacré, French contemporary poetry, gesture, everyday life, words, life.

Sommaire: Introduction. James Sacré et la poésie contemporaine. Le parcours poétique de James sacré. Une écriture de gestes de vie : Interroger, produire, partager, rappeler, regarder, transcender, donner, remercier. Conclusion.

\section{Referencia normalizada}

Fernández Erquicia, I. (2016). "James Sacré: une écriture de gestes de vie ». Thélème. Revista Complutense de Estudios Franceses, Vol. 31, Núm. 1: 107-128. http://dx.doi.org/10.5209/rev_THEL.2016.v31.n1.51599

\section{Introduction}

Dans le panorama poétique français contemporain, il semblerait que certains poètes, par différentes voies et en faisant emploi de différentes méthodes et procédés poétiques, tentent de récupérer une certaine expression de la parole poétique et une remise en place du sujet poétique et, même s'il est vrai que la poésie contemporaine se caractérise par une absence d'écoles ou de groupes poétiques proprement dits, cela ne veut pas dire que certains poètes actuels ne partagent pas quelques intentions ou volontés poétiques communes.

James Sacré, né en 1939 en Vendée, est un poète qui reste auprès du réel, de l'insignifiant même, prenant comme matière créatrice le quelconque, intégrant dans ses poèmes les éléments les plus simples, les plus quotidiens et revendiquant la banalité et l'ordinaire comme l'une des bases de son faire poétique. Ainsi, il s'éloigne d'une conception poétique de l'idéalité, dans laquelle les mots représenteraient l'absolu. Le poète s'éloigne ainsi d'une poésie qui risquerait de tomber dans l'excès, et reste toujours du côté de la lisibilité : «Dans ma poésie j'ai tendance à aller vers les choses plutôt pauvres, mièvres ou maladroites pour défaire ce qui est rutilant ou trop sonore » (Laplace, $1984: 8$ ).

C'est pourquoi nous pouvons présenter James Sacré comme étant un poète qui, tout en voulant récupérer la valeur de la parole poétique, n'hésite pas à prendre comme point de départ de sa poésie la réalité la plus banale et la plus quotidienne pour lui conférer une nouvelle existence langagière, une nouvelle destinée poétique qui lui permet de remettre en valeur cette réalité qui semblait quelconque, et qui revendique la valeur évocatrice et extraordinaire de la réalité et du quotidien les plus anodins. Néanmoins, il faudrait dire que cette démarche entreprise par James Sacré n'est pas exclusive de sa création poétique, et qu'elle concerne d'autres poètes contemporains, tel que le poète et critique Jean-Michel Maulpoix l'affirme dans cet extrait d'une conférence donnée à l'Université de Toulouse le Mirail :

La poésie moderne a tendance à étendre ou à aggraver la part du banal dans l'écriture, par souci de se rapprocher au plus près du réel. La poésie moderne ne se contente pas de puiser une part de son inspiration au plus près de la vie courante, elle tend aussi à faire du commun sa nourriture, à 
faire de l'ordinaire son territoire d'élection. C'est comme ça que je suis amené à employer cette expression, «lyrisme du trivial ». (Maulpoix, 2012).

Cet article tentera d'étudier l'un des procédés que nous avons retrouvé dans l'œuvre poétique de James Sacré et qu'il semble utiliser pour rétablir la présence du sujet poétique sur la page, mais aussi pour revendiquer la réception de l'énonciation poétique par le lecteur : c'est la reproduction de gestes poétiques, qui apparaissent sur la page comme des représentants des gestes de vie du poète. Ce procédé a son origine dans ce qui constitue une dimension indissociable de la poésie de ce poète : le lien entre la vie et les mots, deux dimensions qui ne font qu'un dans la poésie de James Sacré. Le geste poétique est celui qui permet au poète de s'inscrire dans le poème, d'en faire partie en tant que sujet énonciateur mais, il est vrai que chaque période poétique a tendance à refléter les penchants qui la caractérisent dans différentes représentations de ce que nous appelons le geste poétique. Par exemple, et en faisant allusion aux propos de Dominique Rabaté dans son œuvre Gestes lyriques (Rabaté, 2013), il affirme que le geste poétique qui caractérise le mieux le $\mathrm{XX}^{\mathrm{eme}}$ siècle dont nous héritons, c'est celui de l'interruption :

Pour la poésie du $\mathrm{XX}^{\mathrm{ème}}$ siècle dont nous héritons, le geste poétique fondamental est celui de l'interruption. Interruption de la ligne mélodique, de l'effusion, du discursif, la poésie est devenue un discours contrarié, une voix problématique ou un chant empêché, une parole qui ne cesse de se couper pour pouvoir renaître (Rabaté, 2013 : 94).

Mais cette conception du geste poétique que Rabaté associe à l'interruption, peut avoir d'autres interprétations, tel que nous pouvons le remarquer dans les propos du poète et critique Jean-Michel Maulpoix qui, en essayant de décrire le travail d'écriture dans son œuvre La poésie malgré tout (Maulpoix, 1996), le définit comme un geste humain :

Cette pratique est ancienne. L'écriture s'enracine dans la nuit des temps. Elle est un très vieux geste humain, très simple et très sobre qui consiste à tracer des signes sur quelque support. Mais ce geste si élémentaire, presque dérisoire, a accompagné l'advenir de l'homme sur cette terre. C'est grâce au travail silencieux des signes que l'être humain s'est reconnu et défini comme tel. L'écriture est consubstantielle à l'humanité dont elle conserve la vraie mémoire (Maulpoix, 1996 : 45).

Ainsi, nous constatons que, lorsque nous parlons de geste poétique, il est indispensable de faire allusion à la notion de sujet poétique, dans la mesure où le geste poétique constitue une représentation mise en place par le sujet poétique au sein du texte et, nous savons que la manière dont le sujet poétique figure dans le poème, représente l'un des principaux questionnements de la poésie. En ce qui concerne la poésie contemporaine, il faut dire qu'elle cherche à s'éloigner du sujet poétique traditionnel qui, dans des périodes précédentes comme le romantisme notamment, ne faisait entendre qu'une seule voix, celle du «moi » du poète, qui se déployait dans le poème en y exprimant toute la force de son individualité. Le poète contemporain, a pourtant compris que la voix du sujet poétique ne peut plus sonner à l'unisson, parce qu'elle est faite de plusieurs. À partir du moment où Rimbaud a 
déclaré que « je est un autre » (Rimbaud, 1871), la poésie contemporaine a cessé de chanter à l'unisson et elle a commencé à chercher la polyphonie, la pluralité dans sa voix. Tel que Dominique Rabaté l'explique dans l'extrait qui suit, la poésie contemporaine voudrait dépasser le «je » pour atteindre la pluralité dans la voix énonciatrice :

Le poème est le lieu de l'expérience d'un dépassement du « je ». Il nécessite qu'il y ait du sujet, plutôt qu'un sujet. Mais ce sujet provisoire, partiel, mobile est excédé. Ce que le poème cherche à atteindre, c'est une parole qui fonde le sujet tout en le dissolvant ou en le généralisant. [...] Le poème est donc le lieu étrange de cette « expérience » du débordement du sujet. Il est donc aussi l'espace où éprouver la naissance à soi du sujet qui doit pourtant mourir à lui, dans un tourniquet paradoxal auquel le poète doit se soumettre, pour faire advenir, à sa place, le poème. On comprend alors par quelle nécessité logique la voix qui porte le poème doit être mobile et plurielle (Rabaté, $2013: 63)$.

Étant donné que le geste poétique peut être représenté de différentes manières sur la page, en dépendant de la conception et du dessein poétique de celui qui l'accomplit, tel que nous l'avons vu dans les exemples préalablement cités, le but de cet article sera d'étudier le lien existant entre les mots et la vie dans la poésie de James Sacré, et la manière dont il exprime ce lien sur la page, à travers des gestes que nous pourrions aussi bien qualifier de gestes poétiques que de gestes de vie, dans lesquels le sujet poétique s'exprime et finit par faire parvenir au lecteur le geste que le poète lui adresse.

\section{James Sacré et la poésie contemporaine}

Pour comprendre la place que la poésie de James Sacré occupe dans le panorama contemporain, il serait nécessaire de faire un bref parcours par les moments clés qui ont précédé et marqué son époque et aussi son faire poétique lui-même. À partir des années 60, des tendances formalistes et expérimentales se sont développées dans la poésie française, tel que le critique Jean-Luc Maxence nous le confirme dans son recueil intitulé $A u$ tournant du siècle: regard critique sur la poésie française contemporaine (Maxence, 2014) :

Des années 1960 à la fin du XX ${ }^{\text {ème }}$ siècle, l'influence du structuralisme de Roland Barthes, de l'étude en profondeur de la linguistique, s'est révélée progressive et bientôt pesante. En peu d'années, le lyrisme devint synonyme de tradition éculée. L'expression de l'émotion fut bannie, la « nouvelle critique » tint le haut du pavé intellectuel. Dans l'après-68, les démarches de laboratoire foisonnent, abondent, notamment avec la fondation de la revue Tel Quel par Marcelin Pleynet et Denis Roche (Maxence, $2014: 28$ ).

Après cette vague formaliste, quelques poètes, principalement à partir des années 80 , ont voulu s'éloigner de ce traitement de la langue qui avait, d'après eux, poussé la poésie au bord du silence et de l'impossibilité à dire, à force d'aborder les mots en eux-mêmes, sans tenir compte du sujet poétique qui les formulait. C'est donc en essayant de s'éloigner de cette stérilité poétique, et de ce qu'ils ont souvent appelé une "poésie de laboratoire », que certains poètes ont voulu récupérer des 
valeurs poétiques qui semblaient oubliées ou délaissées. Ces mots du poète James Sacré, résument très bien cette volonté de s'éloigner de la tendance expérimentale de la poésie pour récupérer la valeur de l'énonciation et de la réception poétique : «Le poème n'est pas un laboratoire mais un lieu de manifestation pour une énonciation qui est la tienne, lecteur, autant que la mienne (si jamais l'énonciation d'un texte peut se rapporter à une personne particulière) ». (Sacré, 2013b : 159).

Mais Sacré n'est pas le seul à avoir refusé et critiqué cette démarche expérimentale qui s'est trop rapprochée, de la froideur et de la stérilité poétiques. C'est le cas de la poétesse Marie-Claire Bancquart qui, elle aussi, critique la tournure scientiste que la poésie a commencé à prendre à partir des années 60 notamment, en soulignant que c'est à cause de cette orientation de la poésie qu'une partie du public s'en est éloigné :

On allait vers une espèce de scientisme. On risquait de perdre la spontanéité, de même que l'interrogation sur le sens (y compris éventuellement le sens absent, négatif) de l'existence concrète, qui est si essentielle à la poésie... [...] Je crois aussi que cette "poétique-poésie de système » a détaché de la poésie en général toute une part du public, qui ne se plaisait pas forcément à lire ou à entendre des textes qui s'interrogent en langage aride sur leur propre pertinence (Bancquart, $2010: 21$ ).

Un autre poète qui semble critiquer la voie purement formaliste, c'est Antoine Emaz, qui refuse les extrêmes, c'est-à-dire qu'il s'oppose à ce qui est exclusivement et purement lyrique, aussi bien qu'à ce qui est purement expérimental, pour revendiquer un rapport équilibré au faire poétique :

Aux deux extrêmes, que je refuse, il y a donc une écriture narcissique déballant son panier de linge sale, «plus ça pue, mieux ça vaut » et une écriture disons expérimentale visant la pure langue, «ça ne sent plus rien, c'est bien ». Cela laisse un espace énorme entre, peut-être $80 \%$ de la poésie actuelle, avec des tentatives de compositions aussi multiples que dans un laboratoire de grand parfumeur. [...]D'une certaine façon, ma critique du clinquant de l'image rejoint celle de l'abstraction formelle. C'est privilégier la langue, la faire ronfler pour elle-même alors qu'à mon avis, elle devrait toujours être subordonnée à vivre, à la tension de vivre (Emaz, 2012 : 84-90).

Face à ce rejet des voies expérimentales et stérilisantes de la poésie, certains poètes ont revendiqué une récupération de l'expression lyrique du sujet poétique, qui pourrait correspondre à ce que Dominique Rabaté appelle des «gestes lyriques », et qu'il défini ainsi :

Interroger, appeler, interrompre, donner, promettre: ces verbes parmi quelques autres, désignent certains des gestes lyriques que la poésie moderne permet d'effectuer selon des opérations de langage qui lui sont propres. [...] Ce sont de tels gestes que vise le poème. Et ce sont eux qui jouent, en amont, un rôle moteur pour alimenter l'énergie lyrique qui en découle (Rabaté, 2013 : quatrième de couverture).

Également, le poète Jean-Michel Maulpoix, qui a profondément théorisé sur la récupération et la reformulation de valeurs lyriques dans la poésie contemporaine, en créant des notions comme le « Nouveau Lyrisme » et le «Lyrisme Critique », 
consacre une partie de son travail à la réflexion sur le geste lyrique, qu'il définit ainsi :

Le geste lyrique est alors par excellence celui de l'offrande et du partage, sans espoir de retour. «La poésie s'adresse à tous en particulier ». «Écrire s'adresse à quelqu'un en qui on ne croit pas, mais qui nous parle avec des mots qui cherchent nos lettres, qui prennent notre voix ». Le lyrisme consiste à accueillir l'anonyme en soi, à atteindre «la crête d'impersonnel » en "épousant la singularité de sa propre cadence » (Maulpoix, $1998: 126$ ).

Néanmoins, il ne faudrait pas confondre cette volonté de récupération du sujet poétique avec un retour en arrière, vers des effusions lyriques, des ornementations du texte poétique et des épanchements personnels plus proches d'autres périodes poétiques comme le romantisme et, sur ce point, James Sacré constitue un très bon exemple qui nous montre qu'une récupération d'un certain souci pour le sujet poétique, pour ce qu'il exprime par le biais des mots poétiques et par ce qu'il fait parvenir au lecteur, est possible, sans pour autant tomber dans un excès ou un débordement lyriques :

Le terrorisme littéraire antisentimentaliste me semble aussi détestable que les dénonciations sentimentales de l'intellectualisme. La poésie je crois se nourrit aussi bien d'émotions que de règles (les deux correspondant à la fois à des formes du langage et à des attitudes de vivants). Il n'y a pas de grammaire «idéale » qui permettrait de mesurer ou de juger ces chevauchements (Sacré, 2013b : 155).

\section{Le parcours poétique de James Sacré}

Dans ce contexte contemporain, l'écriture de James Sacré est marquée par un prosaïsme qui s'intéresse au quotidien, à l'ordinaire, à la vie de tous les jours.

James Sacré passe son enfance et son adolescence dans la ferme de ses parents, en Vendée, où il est en contact aussi bien avec le patois qu'il entend et parle à la maison, qu'avec le français qu'il apprend sans aucune difficulté à l'école. Voilà probablement pourquoi il n'hésite pas à faire entrer le patois de son enfance dans ses poèmes, qui sont écrits en français, mais qui laissent toujours une petite place à des expressions et même des mots de ce patois, parfois marqué même par des tournures linguistiques propres à l'enfance. De même, l'introduction du patois dans le poème, provoque parfois que la syntaxe soit un peu déformée ou détournée de son emploi conventionnel :

Maman disait la berne. Va chercher une berne!

Va-t-en cri une barne qu'aurait dit mon père (Sacré, 2010a : 35).

En 1965 il part aux États-Unis où il fait des études de lettres et enseigne ensuite dans une université du Massachussetts. Pendant son séjour aux États-Unis, il fait des voyages en France, en Tunisie, en Italie et au Maroc et, actuellement, il vit à Montpellier. Tous ces voyages, ces contacts avec d'autres langues étrangères marqueront l'auteur et son œuvre et surtout son rapport à la langue, sa manière d'accéder aux mots et de tester leur sonorité. Le poète avoue même que le fait de 
parler une langue autre que le français, l'a encouragé à interroger et à mettre en question la langue française, rendant ainsi sa manière d'aborder et d'interroger la langue très personnelle et n'hésitant pas à développer un regard critique et de mise en doute vis-à-vis de la langue qu'il a choisie pour véhiculer sa poésie. D'après lui, c'est le fait d'avoir fréquenté le patois et aussi d'autres langues étrangères comme l'anglais des États-Unis, qui aurait poussé l'auteur à désirer le français comme une langue où il pourrait faire un autre emploi des mots et trouver son identité poétique, en même temps qu'elle représenterait un espace de quête, de questionnement et de critique de la langue :

Le patois et la langue étrangère interrogent le français bien établi, sûr de lui, avec ses hiérarchies. Si le patois est une langue étrangère par rapport au français, je peux dire que ma fréquentation très malhabile et très approximative des langues étrangères m'a conduit à désirer une autre langue, qui est ma langue de poète où tout d'un coup, je serais dans un espace plus libre (Guichard, 2006 :1423).

D'ailleurs, il ne faut pas oublier que l'une des caractéristiques du poète contemporain, c'est qu'il accompagne son travail de création poétique d'une activité de critique, tel que Daniel Lewers nous le montre dans son œuvre Introduction à la poésie moderne et contemporaine (Lewers, 2010) :

Depuis Baudelaire, et pour reprendre la pertinente formule de son étude sur Richard Wagner: «Tous les grands poètes deviennent naturellement, fatalement, critiques ». [...] Dès la seconde moitié du XIX ${ }^{\text {ème }}$ siècle la poésie française est de plus en plus devenue une métapoésie avide d'être une réflexion sur elle-même ainsi que sur les poétiques qui l'ont précédée ou qui lui font simplement escorte (Lewers, $2010: 1$ ).

Cette nouvelle tâche de critique que le poète contemporain semble accomplir, lui permet d'aborder l'utilisation de la langue et la présence du sujet poétique dans sa poésie, à partir d'un regard méfiant, qui le conduit à mettre en question son propre travail de création poétique, tel que Rabaté le constate dans l'extrait qui suit : «Le sujet lyrique, compris comme capacité de chant et d'enchantement, comme puissance mélodique et mélodieuse, se voit entravé par un sujet critique qui le dédouble pour en faire un sujet de doute, de l'ironie et de la distance » (Rabaté, $2013: 94)$.

James Sacré a commencé son parcours poétique dans les années 60, et il écrit toujours, complétant ainsi sa déjà vaste bibliographie, composée aussi bien d'ouvrages poétiques que de travaux de critique littéraire. Associé d'un côté au penchant lyrique de la poésie et, de l'autre, à un côté plus littéraliste, James Sacré évite les classements et se considère un poète spontané qui ne suit aucune tendance concrète. En tant que poète contemporain, Sacré n'aime ni les classifications ni les étiquettes et préfère travailler suivant des instincts poétiques et non pas en essayant de trouver une place déterminée dans le panorama poétique contemporain. C'est pourquoi, lorsque les critiques littéraires le placent dans une démarche qui se situerait entre littéralité et lyrisme, il refuse de se limiter à cet entre-deux qui ne sert pas à qualifier son œuvre poétique dans toute son ampleur et dans toute sa richesse : 


\begin{abstract}
Non, je n'ai voulu me placer nulle part. Je n'aime pas trop « entre littérarité et lyrisme », [...] Il n’y a pas pour moi d'écart entre littérarité et lyrisme. On pourrait dire que la littérarité est davantage un sentiment de la matérialité de l'écriture, des mots, d'une phrase et que le lyrisme, c'est peut-être comme pouvoir mettre dans ces mots un désir d'autre chose qui dépasserait ces mots ou serait derrière. Mais ça finit par se rejoindre. Je n'ai jamais eu le désir de trouver ma place, mais il se trouve que dans mes façons d'écrire, des gens très divers ont trouvé quelque chose qui leur plaisait (Guichard, $2006: 14-23$ ).
\end{abstract}

C'est vrai que James Sacré s'est souvent approché d'une certaine émotion que l'on pourrait qualifier de lyrique dans ses poèmes, mais la manière dont il aborde cette émotion est toujours très timide, complètement éloignée des dramatismes et des ornementations excessives et garde toujours une certaine distance par rapport à l'expression purement émotive, grâce à une mise en question constante de la langue en général, et à un refus des débordements lyriques plus précisément. On remarque dans son écriture une volonté de retrouver une parole équilibrée et, surtout, dépassionnée, ce qui conduit le poète à refuser le langage beau et élaboré. Sacré s'exprime comme tout le monde, en évitant les mots pompeux et emphatiques. Nous constatons également qu'il n'hésite pas à critiquer les poncifs du lyrisme, pratiquant ainsi ce que nous pourrions appeler un lyrisme non-ardent ou nonenthousiaste.

Écrivant en vers ou en prose indifféremment, James Sacré avoue que le poème reste poème quelle que soit la forme choisie pour le mettre sur la page, et c'est plutôt ce que le poème veut dire qui représente un défi pour le poète :

Je ne fais vraiment pas de différences entre le vers et la prose. Sauf une petite différence technique impossible à définir. Je suis incapable de me souvenir comment je suis passé du vers à la prose. C'est la même chose. Prose ou vers, c'est du poème de la même façon. Ce serait effectivement un calcul ou une ruse de vouloir utiliser le vers lorsqu'il s'agit d'évoquer quelque chose d'un peu trop intime, mais ce n'est pas ma position. Le problème c'est plutôt qu'on est dans l'ignorance de ce qu'on veut dire, l'ignorance de ce que l'on dit et le sentiment que si on savait exactement ce qu'on veut dire on n'y arriverait pas (Guichard, $2006: 14-23$ ).

\title{
Une écriture de gestes de vie
}

La poésie de James Sacré cherche une relation constante entre la vie et les mots, entre l'expérience vécue et ce que les mots peuvent en dire. Et, puisque le poète vit et a une expérience vécue, il y a toujours quelque chose à dire, quelque chose à écrire, aussi simple, banale ou anodine cette expérience soit-elle parce que, pour James Sacré, tout est digne de devenir de la matière poétique. Nous pourrions aller plus loin encore, en affirmant que c'est précisément cette volonté de créer de la poésie à partir des événements les plus quotidiens, qui permet au poète de rester au plus près du réel, en contact étroit avec la vie, évitant de s'évader vers des dimensions poétiques chimériques. Chez Sacré, le regard porté sur le quotidien part toujours de l'expérience vécue ou du souvenir de cette expérience, donc, si le poète prend la peine de regarder les choses les plus banales et les plus ordinaires du quotidien, c'est parce qu'elles ont un rapport indéniable avec ce vécu-là. C'est à 
travers ces petits détails qui font partie de la vie de tous les jours que Sacré parvient à mettre du vécu sur la page.

En faisant le lien entre la volonté de Sacré de mettre en rapport le vécu et les mots poétiques, et son désir de transférer ce rapport sur la page, nous parvenons inévitablement à la notion de geste poétique qui a déjà été annoncée, comme étant un procédé caractéristique de l'œuvre poétique de James Sacré, qui lui permet d'aborder la création poétique à partir de différents gestes qui deviennent à leur tour des formules énoncées par le sujet poétique au sein du texte. Nous aborderons donc notre étude à partir de la mise en place de différents gestes poétiques qui permettent au poète d'accomplir diverses actions sur la page, des actions que nous réduirons à des verbes comme interroger, produire, partager, rappeler, regarder, transcender, donner et remercier, qui déterminent les différentes actions que le sujet poétique pourra effectuer sur la page à travers la mise en place de gestes poétiques, et qui finissent tous par renforcer le lien indéniable que la poésie de James Sacré semble entretenir avec le vécu.

\section{Interroger}

Pour James Sacré, l'écriture représente une manière d'interroger son expérience de vie pour pouvoir la partager avec autrui, avec le lecteur, en la faisant passer par la dimension des mots poétiques, voilà pourquoi le premier geste poétique que nous étudierons est associé au verbe interroger, car, pour questionner son vécu, le poète a besoin de gestes d'écriture : des gestes qu'il produira avec sa main, avec sa plume, et qui pourront traduire en mots les gestes de vie qui sont à l'origine de tout acte d'écriture, lui permettant ainsi d'effectuer tout un travail de questionnement aussi bien sur le travail de création poétique que sur le vécu qui l'inspire.

L'écriture de James Sacré veut donc mettre en place une écriture de gestes de vie, sa poésie est faite de gestes et c'est ainsi qu'il atteint une écriture qui est indissociable de la vie, aussi ordinaire ou banale soit-elle. D'ailleurs, dans cette réflexion qui concerne aussi bien les gestes de vie que ceux d'écriture, James Sacré finit par se demander s'ils ne constituent pas, en définitive, un seul et unique geste. Donc, James Sacré se laisse atteindre par les mots, qui touchent le poète par leurs gestes. Il est conscient de la nature éphémère et difficile à saisir des mots, mais il ne cesse pas pour autant de croire en leur pouvoir d'atteindre le poète et de lui transmettre tout leur sens à travers leur gestuelle :

[...] Les mots ça a pas d'odeur

(ni merde un peu ni sueur, pas non plus l'odeur

du propre). Est-ce que pourtant les voilà pas qui te touchent? (Sacré, $2000: 14$ ).

Cet extrait que nous venons de citer représente un exemple de l'importance de l'oralité dans la poésie de Sacré, qui n'hésite pas à utiliser des tournures courantes et qui sont typiques d'un emploi oral et familier du langage. Par exemple, ici, nous remarquons l'absence de la particule «ne» de la négation, qui constitue une marque typique du langage oral. Également, l'emploi de parenthèses est un autre 
élément très fréquemment utilisé par Sacré, et qui apporte des ruptures et des suspensions dans le rythme, ce qui nous rapproche ainsi du débit oral de la langue. Cet emploi du rythme oral de la langue, permet en plus au poète de refléter le rythme de la vie dans le rythme de l'écriture, ce qui montre que vie et poème ne font qu'un chez James Sacré, et que c'est le geste du poète qui permettra de les réunir sur la page, les offrant ainsi, dans un dernier geste de don poétique, au lecteur.

Ainsi, et en ce qui concerne ce premier mouvement d'interrogation qui apparaît dans le texte en tant que représentation du geste poétique, nous pourrions dire que le questionnement que Sacré met en place à travers les gestes d'écriture en poésie, porte aussi bien sur la vie elle-même que sur le fait poétique, un double questionnement qui demeure condensé dans le geste poétique que Sacré effectue au sein du texte lui-même. C'est-à-dire le principal travail de la poésie, c'est de questionner la vie, tel que le poète Antoine Emaz le manifeste dans ce passage : « [...] pour l'heure, il suffit de faire son travail [le travail de la poésie], c'est-à-dire d'entrer ou de rester dans un perpétuel questionnement de vivre » (Emaz 2009 : 92). Même s'il est vrai que ce questionnement sur la vie conduit inévitablement le poète, notamment dans la poésie contemporaine, à s'interroger sur la poésie elle-même, tel que le poète et essayiste Jean-Claude Pinson l'explique: «Elle [la poésie] s'inquiète de son rapport à la langue, en privilégie souvent, même, le questionnement, la poésie (celle du moins qui cherche et se cherche) est le cœur vivant de la littérature, son noyau productif » (Pinson, $2002: 12$ ).

\section{Produire}

Une fois que le poète a interrogé le vécu et le fait poétique, il s'adonne à la tâche de la création poétique elle-même, un travail que nous avons associé au geste poétique dont l'action consiste à produire. Chez Sacré, le travail de production écrite est souvent décrit comme un travail fait à la main, un travail qui permettra de reproduire les gestes de vie. Par exemple, dans cet extrait du recueil Si peu de terre, tout (Sacré, 2000), le travail d'écriture est associé à la tâche de faire la lessive : c'est un travail de gestes, un travail fait à la main, que le poète compare à sa tâche de création poétique. De même, en essayant de qualifier ce travail d'écriture sous l'apparence de lessives, l'auteur désigne son écriture comme étant une écriture courte et qui donne la sensation de s'écrire toute seule, comme si ces gestes d'écriture étaient presque involontaires chez le poète :

Écrire autour de ces linges c'est comme un travail de lessive à faire. Forcé d'y revenir à la fin, mais le poème réduit tout à presque rien. Que des petits gestes; au fond j'ai jamais fait de lavage en grand. Courtes lessives dans les lavabos d'une école ou de la caserne, bassines qu'on laissait tout tremper dedans longtemps comme si ça allait laver tout seul (Sacré, 2000 : 15). 
En faisant allusion aux propos du poète et critique Jean-Michel Maulpoix, l'écriture poétique est toujours à la recherche du geste poétique, un geste simple qui ne voudrait que parvenir à poser la plume sur la page, c'est-à-dire à produire à la main des mots qui seront posés sur la page :

L'écriture est une affaire de main. Un écrivain est un homme qui pense avec la main, au gré de tracés et de ratures. [...] Au chant qui vient par le souffle, la bouche et le pas du marcheur lyrique, s'oppose un travail d'homme penché, immobile face à la page d'écriture : des mots lui viennent en lignes dans la main. [...] L'écriture est à la recherche de ce geste étrange et simple : le toucher d'une plume sur la page blanche (Maulpoix, 2005 : 97-99).

Dans cette même lignée qui veut associer l'écriture poétique à la production d'un geste qui demande l'implication du mouvement de la main, d'un geste physique et actif qui puisse témoigner de la présence du sujet poétique sur la page, Dominique Rabaté associe la poésie à la peinture, et avoue que la poésie convoite l'immédiateté du geste du peintre, qu'il aimerait bien atteindre par le biais des mots :

Quand il rêve d'une efficace de la parole, d'une sorte de performativité qui peut toucher à la magie, le poète se tient dans le proche voisinage du peintre. Au plasticien, il voudrait reprendre l'effet immédiat d'un geste qui trace, peint, entaille, rend visible. Du geste physique à la trace produite, c'est le mouvement même qui ordonne le trait. Le geste créateur, jusque dans son tremblé ou ses ratés, passe par le corps, par l'impulsion ordonnée d'une pratique. L'écrivain semble voué à une tâche plus cérébrale, puisqu'il est « conditionné » par l'univers des signes, par les mots, par le « verbal » dont il aspire, paradoxalement, à sortir par le moyen même du langage (Rabaté, 2013 : 227).

Dans cette conception du geste poétique en tant que production manuelle de la parole, l'origine paysanne de James Sacré est très présente en ce qui concerne sa conception de l'écriture. Nous voyons par exemple qu'il associe souvent le travail du paysan, ce travail dans les champs, avec les animaux, etc. avec le travail de l'écrivain, qui devient une sorte de laboureur des mots. C'est donc à nouveau par les gestes, des gestes de production cette fois-ci, que James Sacré rapproche l'écriture de la vie. Dans ce cas-là, ce sont les gestes du paysan dans son travail qui sont mis en rapport avec les gestes d'écriture que le poète entreprend lorsqu'il est confronté aux mots :

[...] C'est vrai aussi que les deux

activités, écrire, cultiver, prennent forme dans la

même argile originelle (toujours là d'ailleurs, nul

secret perdu),

[...] Mais quand je prends

de l'encre et du papier (ou mon ordinateur, avec

ses bruits et ses couleurs)

Qu'est-ce donc que je laboure ? (Sacré, 2000 : Quatrième de couverture).

Dans le recueil intitulé Les mots longtemps, qu'est-ce que le poème attend? (2003), il y a un fragment intitulé "Un brabant double avec des voiles », dans lequel c'est comme si le poète était le paysan qui prend le brabant, cette machine 
métallique à labourer, et la faisait tourner avec des mots, pour enfin aboutir à la création d'un poème, reproduisant ainsi le geste du paysan sur la page et avec le brabant propre au poète, c'est-à-dire, sa plume. En plus, le poète parvient à reproduire le mouvement de la machine à labourer avec la forme du poème, qui semble tourner en boucle comme la terre sous les fers de la machine ; ainsi, le geste de la machine qui fait tourner la terre, devient le geste poétique qui fait tourner les mots :

\begin{abstract}
Mouvement l'encre et le papier le vent ça parle de fontaine et de ce n'est rien poème soudain qui se déprend la rime est un buisson percé le cœur il sèche dans son mot cœur le ce n'est rien mouvement l'encre le temps dans la lumière je la vois l'été la silence dans les prés j'aime la solitude le temps mal mesuré.
\end{abstract}

Mouvement l'encre
le cœur est lent
que l'été silence

Voilà mouvement l'encre et le papier le vent
Me parle de fontaine et de la référen-
ce n'est rien poème qui soudain se déprend
La rime est un buisson percé le cœur est lent
Il sèche dans son mot cœur le vent le balan
Ce n'est rien voilà mouvement l'encre et le temps
(dans la lumière que je vois et l'infinie quantité de bleu
que l'été silence dans les prés les visages que j'aime sont
de la solitude et du temps qui n'est pas mesuré) (Sacré, 2003 : 161).

Cet extrait nous montre également que Sacré est prêt à utiliser une syntaxe volontairement simple et près de l'oralité, qui le conduit parfois à la rudesse dans le ton. Également, nous voyons que les variations typographiques sont utilisées ici, mais toujours au service du signifié, c'est-à-dire la forme du poème, avec ses sauts à la ligne, ses parenthèses et ses coupures et irrégularités syntaxiques et rythmiques, cherche à renforcer ce que le poème dit, comme dans ce cas-là, où la distribution des mots nous rappelle le mouvement de la machine à labourer, renforçant ainsi le lien indéniable qui existe entre le vécu et les mots poétiques dans la poésie de James Sacré. De même, il serait important de souligner que les brusqueries syntaxiques présentes dans le poème, ainsi que des anacoluthes, ne sont pas des maladresses de style, mais des tournures que le poète met en place délibérément, pour ainsi s'éloigner des emplois conventionnels et souvent usés du langage.

Dans cet autre poème qui suit, le monde paysan qui a entouré l'auteur pendant son enfance et le poème sont mis en rapport. Nous y voyons toujours cette alliance inexorable entre les gestes du vécu, le souvenir de ce vécu et sa mise en mots par le langage poétique, toujours en cherchant d' " ameuter l'écriture » : 
Il faudrait parcourir ce poème par le biais d'une grande écriture d'avoine jetée,

qu'elle provienne de l'existence d'une paille longue (je me souviens de ce geste qu'on faisait pour choisir le lien des gerbes, comme d'en peigner la paille).

Une avoine jetée par le travers de la page et toute la figure des panicules mise dans un bel ordre d'éventail.

L'enfance y fut belle et dès lors toute sa légèreté d'oiseaux morts et secs parmi les herbes sèches.

Parcourir par le biais des chaumes un présent pour ameuter l'écriture,

et la jeter, mourir et ramasser les oiseaux dans les poèmes (Sacré, $2003:$ 143).

\section{Partager}

Dans la mise en page des gestes poétiques, Sacré n'hésite pas à recueillir les gestes de vie d'autrui dans son poème, des gestes qui, en principe, ne lui appartiennent pas, mais qu'il intègre dans son poème parce qu'ils ont aussi fait partie de sa vie, même si d'une manière indirecte, à savoir à travers le contact que le poète aurait pu avoir avec d'autres personnes. Nous parlons donc de partage dans ce cas-là, dans le sens où Sacré n'hésite pas à partager l'espace poétique avec autrui, il se décide à faire entrer autrui dans la dimension poétique, en accomplissant ainsi un geste poétique qui lui permet de partager la page. Ainsi, en intégrant autrui dans l'espace poétique, Sacré fait preuve d'une tendance présente dans la poésie contemporaine qui consiste en la quête de l'altérité et de la multiplication des voix poétiques, qui nous permettra en même temps d'atteindre une meilleure connaissance du " moi », tel que Maulpoix l'explique dans cet extrait de son recueil La poésie comme l'amour (Maulpoix, 1998) : «Mais l'altérité n'est pas réductible à l'impersonnalité : elle est aussi la découverte en autrui, par le sujet, de sa propre incompréhensibilité. Le lyrisme découvre le "moi" par le détour de l'autre et oscille ainsi sans cesse entre l'étrange et le familier, le propre et l'anonyme » (Maulpoix, $1998: 126$ ).

Parmi les présences d'autrui que Sacré accueille dans son poème, il faut dire que la figure du père apparait souvent. Sacré semble avoir intégré les gestes de celui-ci en les faisant entrer dans ses poèmes et, de cette manière, le poème représente un espace qui permet de montrer et de garder les gestes du poète, mais aussi de partager cet espace avec les gestes de ceux qui ont fait partie de sa vie, que le poète garde en lui et met sur le papier pour pouvoir les conserver. Nous constatons ici une volonté de la part du poète de maintenir les souvenirs - ou les gestes pourrions-nous dire - vivants, et c'est l'espace poétique qu'il a choisi pour ce travail de conservation, comme nous le voyons dans le fragment qui suit :

Deux trois morilles mon père les tirait de son pale- 
tot il avait passé par quels fonds de prés buissons vieux talus solitude ? Je l'entends marcher dans les herbes ronces que sa main écarte je veux avoir ses gestes présents dans mes poèmes j'en reviens comment deux trois mots vite où les as-tu ramassés ? (Sacré, 2012b : 46).

Dans cet extrait que nous venons de citer, le rythme oral est aussi très marqué, notamment par l'absence presque totale de signes de ponctuation, à l'exception de deux signes d'interrogation, dont la présence est mise en relief par le fait qu'ils constituent le seul signe de ponctuation du poème.

Le poète avoue qu'il se nourrit des gestes de son père aussi bien que des lectures faites et des mots trouvés dans des dictionnaires, ce qui montre que les gestes de vie, aussi bien que les mots, constituent de la matière créatrice pour James Sacré, et qu'il est prêt à les rassembler tous dans un même espace de partage poétique, qui est le poème lui-même. Le monde des mots est également associé au monde paysan, puisque lire et écrire sont des activités qui ont des points en commun avec le travail de labourer : il s'agit de tâches quotidiennes où l'on ne trouve pas de réponse aux grandes questions, mais qui, du moins, nous montrent l'obscurité du monde. Ce fragment représente un très bon exemple pour comprendre que la poésie de Sacré est une poésie qui, malgré le fait qu'elle prend ses appuis dans la banalité du quotidien, elle est capable d'atteindre la transcendance et d'accompagner le poète dans le questionnement sur l'existence qui le caractérise :

Il y a eu ces gestes de mon père, mais sans doute aussi des livres que j'avais lus, ou pas encore, les mots dépris des dictionnaires [...] lire est-ce que c'était pas du coup labourer nulle part peutêtre, seulement rêver [...] m'en aller pour je saurai jamais où, quitter perdre (et sans rien gagner par ailleurs) le va-et-vient répété du vrai travail tous les jours, fallait bien continuer, sans réponse à rien, ni à des questions mal formulées ni à d'incertains désirs, mais travail qui montrait du moins la très proche obscurité du monde (Sacré, 2003 : 155-156).

Également, cet extrait nous permet d'affirmer que, même lorsque Sacré écrit en prose, il maintient le même ton près de l'oralité que nous avons remarqué dans d'autres extraits en vers.

\section{Rappeler}

La mémoire est un élément très présent dans la poésie de Sacré, dans la mesure où elle représente le réservoir du vécu du poète, et c'est elle qui conduit le poète à accomplir un autre geste poétique sur la page, un geste qui cherche à rappeler, à récupérer des souvenirs qui seront transposés à la dimension poétique à travers la mise en place de gestes poétiques. Par exemple, nous voyons que l'auteur parle des souvenirs d'enfance, des moments qu'il a passés avec son père qui lui a appris des gestes et des mots qui sont, à la suite, devenus des gestes à lui et qui ont donc atteint ses poèmes. Encore une fois, nous voyons que les mots poétiques chez Sacré sont issus du vécu, ou sinon, des souvenirs de ce vécu, et sont mis sur la page à travers des gestes poétiques qui permettent de les rappeler : 
Naturellement je suivais mon père à la chasse. Estil devenu pour moi comme un fusil qui marche ? Nous apprenions des oiseaux mais surtout le silence et des gestes dans un paysage.

Lieux-dits mots que mon père m'a montrés (fonttaine et fusil) j'aimerais savoir qu'ils sont seulement la violence mais la tendresse de son cœur. Du mien. Pour ne pas avoir peur des souvenirs ni des poèmes qui sont vides (Sacré, 2003 : 185).

D'après cet autre fragment, James Sacré aurait découvert le rapport entre les mots et les gestes, c'est-à-dire, entre le langage et le vécu, pendant son adolescence. Nous voyons donc que l'enfance et l'adolescence sont restées des périodes très marquantes dans la vie du poète, qui semble avoir découvert la mise en gestes de ce rapport entre le langage et la vie pendant son adolescence; une découverte qui marquera son œuvre poétique à jamais :

La découverte de l'alliance secrète des mots et des gestes est venue avec l'adolescence quand les prairies signifièrent des fêtes, ou des femmes (Sacré, 2003 : 141).

D'ailleurs, d'après Sacré, cette période qui fait partie de la vie de tout être humain, l'enfance, resterait en nous dans nos gestes présents ou actuels, ces gestes qui, après, deviennent des gestes d'écriture dans l'œuvre de James Sacré :

Le plus infime pays d'enfance est-il pas

Toujours naissant

Et toujours qui s'efface

En nos gestes d'aujourd'hui ? (Sacré, 2013 : 144).

\section{Regarder}

Le poète regarde le monde qui l'entoure en accomplissant ainsi le geste poétique qui concerne l'action de regarder, mais il s'agit d'un regard particulier, ce n'est pas le regard conventionnel que nous pourrions faire avec les yeux, mais c'est un regard que le poète accomplit grâce à l'utilisation des mots poétiques. C'est-à-dire le poète porte son regard particulier sur la réalité environnante et fixe ce regard dans le poème, à travers la mise en place du geste poétique.

En rapport avec le geste poétique qui permet au poète de porter son regard sur la réalité, il y a une autre figure qui apparaît de manière récurrente dans l'œuvre poétique de James Sacré, et qu'il utilise pour caractériser ou définir les gestes d'écriture en rapport avec le regard qui sont mis en place par le poète : c'est la figure du photographe. Souvent, nous voyons que le travail du photographe est comparé à celui de l'écrivain, parce que tous deux tentent de figer quelque chose sur un support (le papier ou le cliché) à travers un geste, poétique ou photographique, mais toujours en l'imprégnant de leur propre regard : 
[...] et c'est pas facile

De comprendre comment le regard de quelqu'un se mêle

À ces cadrages qu'oriente si fortement

Ce qu'il a photographié. Je pense

À la façon qu'a de progresser mon poème

Entre le bruit des mots que j'emploie et forcément

Du sens qui me vient

Dans leur mise en forme (et leur brouillage malgré un désir

de clarté)

Sur le papier (Sacré, $2010: 45$ ).

Ainsi, Sacré définit la photographie toujours en faisant le lien avec l'écriture, parce qu'il sait que les deux disciplines permettent de fixer et de transposer le regard sur un support qui pourra l'accueillir et le garder, que ce soit à travers l'exécution du geste poétique ou du geste de prise de la photographie :

\author{
Lui qui prend des photos, en parle-t-il \\ Dans ce qu'il donne à voir? \\ Les couleurs, les formes qu'il affirme \\ Ne disposent pas de mots \\ (sauf à en suggérer par des arrangements de figures, \\ Ou par une insistance à reprendre certains motifs) \\ C'est que discours de gestes muets (Sacré, $2010: 49$ ).
}

Le regard devient le point commun qui unit le faire du poète avec celui du photographe : le premier regarde à travers les mots, le deuxième à travers la visée de son appareil, mais, en définitive, tous deux transforment ce regard en gestes qu'ils tentent de fixer pour éviter qu'ils tombent dans l'oubli et la disparition. Les mots serviraient donc aussi à voir, on pourrait dire qu'ils sont les yeux du poète : «Comment tu regardes avec ton poème ? » c'est le titre d'un des poèmes du recueil Tissus mis par terre et dans le vent (Sacré, 2010a), dans lequel l'auteur se pose la question suivante : «[...] les mots pour voir ? » (Sacré, 2010a : 121-122). De même, le poète se demande si la beauté des objets est visible avec un simple coup d'œil, ou s'il faut un geste artistique, celui du poète, ou celui du photographe, pour la mettre en valeur :

À cause que posées contre les murets bas

Sont-elles, par leur seule présence, de la beauté ?

Ou s'il y faut le cadrage d'une photo

Ou les mots (pas forcément ceux que voilà dits)

D'un poème?

Vieille question sans doute qui restera sans réponse

Mais c'est pas désagréable, ni même frustrant, d'y penser (Sacré, 2010a : 23).

Que ce soit à travers les gestes propres au poète ou ceux d'autrui - comme les gestes de son père que le poète tente d'intégrer dans sa poésie - ce qui est clair c'est que chez James Sacré l'écriture est associée au geste, toujours issu du vécu, et cette 
association représente une manière de nous rapprocher du langage dans toute sa potentialité, tout en restant en contact avec l'expérience de vie :

Dans ces courtes embardées d'écriture (je

les voudrais comme une joue surprise, comme

un geste qu'on allait faire) faudrait se jeter

pareil qu'autrefois $[\ldots]$

Des mots qui sont pourtant très rouges sem-

blent disparaître au fond de la phrase. À force

d'écrire on voit surtout la trame du langage (Sacré, 2000 : 98).

Ainsi, nous constatons que les gestes de vie constituent le matériau d'écriture pour l'auteur :

Je décris que des façons d'arranger des phrases

À cause de ces matériaux divers : des notes sans projet, les

gestes d'une vie [...] (Sacré, 2010b : 10).

\section{Transcender}

Il y a un autre aspect dans l'association entre gestes de vie et gestes d'écriture qui fascine le poète et le pousse à exprimer sa fascination sur la page : c'est le rapport étroit qui existe entre l'immensité de l'univers et l'exigüité des gestes quotidiens qui rend le poète perplexe et le pousse à mettre ce rapport par écrit. Le poète, qui n'hésite pas à faire entrer dans la poésie les faits les plus anodins du quotidien, est frappé par la possibilité de pouvoir conserver dans sa poésie un certain esprit de transcendance, né du lien éphémère mais insistant entre les gestes de vie les plus insignifiants et l'immensité de l'univers qui nous entoure, ce qui montre que l'accomplissement du geste poétique peut atteindre un certain niveau de transcendance au sein du texte poétique :

Fugitif rapport entre l'immensité du ciel dans une attente

Et le temps d'un geste pour tenir un caillou dans mon

cœur (Sacré, 2010b : 340).

Une fois que le poète a découvert la puissance des mots poétiques, capables de reproduire ce lien permanent entre la vie et l'écriture, et d'atteindre ce que nous pourrions qualifier comme une transcendance poétique, il entame une démarche dans laquelle il ne perd pas l'espoir de «mettre la main » dans les mots, comme nous le voyons dans ce fragment du recueil intitulé Les mots longtemps, qu'est-ce que le poème attend? (Sacré, 2003) et dont le titre est repris par Sacré dans sa réflexion sur la possibilité de s'approcher des mots par le biais de gestes :

Dire que le poème attend, dire qu'avant d'être écrit il y a eu les mots longtemps, ça permet au moins ce titre que j'aime bien, mais c'est surtout parler par métaphores, ou par le moyen de métonymies telles distendues ! c'est bien seulement moi qui attends à travers mon poème, moi qui ai longtemps, d'abord, regardé des mots... pas vraiment pour les apprivoiser, mais pour m'en approcher un peu, à l'occasion les caresser, pour les entendre mieux, et parfois, je l'ai cru, mettre 
la main dedans. La main plutôt que les yeux parce qu'en général on n'y voit pas tellement clair dans les mots (Sacré, 2003 : 198).

James Sacré est sûr que son travail de poète n'est en réalité qu'une reprise incessante du même geste : celui d'écrire un livre de poèmes. Et, même si quand il finit un recueil, il a la sensation de passer à autre chose, il sait que tout ce qu'il a écrit, et tout ce qu'il a lu aussi, l'accompagneront toujours, parce que tout ce qui passe par la plume du poète, tout ce qui touche la page grâce à l'accomplissement d'un geste poétique, demeure dans l'expérience vitale du poète et parvient ainsi à transcender : «En fait, je ne suis jamais passé à autre chose : je ne fais toujours que reprendre mon geste : écrire un livre de poèmes » (Sacré, $2003: 223)$. Le poète va plus loin encore et rêve de devenir un livre, un livre inachevé comme une vie est toujours inachevée, mettant, une fois de plus, l'écriture et la vie au même niveau : «[...] des poèmes dans lesquels se désarroit mon désir d'être un livre. Un livre qui se continue, donc, depuis les premières pages écrites et qui ne sera jamais vraiment terminé. Comme une vie n'est jamais terminée : mourir empêche toujours qu'on termine » (Sacré, $2003: 224)$.

\section{Donner}

Néanmoins, une fois que le poète est parvenu à reproduire les gestes de vie dans le poème grâce à l'écriture, il lui reste un dernier geste à accomplir : celui de l'offrande, du don poétique, nécessaire pour partager le poème avec le lecteur. Pour James Sacré, chaque poème est un geste pour rencontrer l'autre, et le dernier geste du poète est celui qu'il fait lorsqu'il dirige ses mots au lecteur: après le geste d'écriture il y a donc le geste du don, le dernier geste du poète et celui qui clôture le parcours de gestes poétiques qui représentent des gestes de vie, entamé par James Sacré :

Je pense que le livre qui s'en va vers d'autres lecteurs, on ne sait pas lesquels, c'est une autre sorte de rencontre. Il doit quand même y avoir une permanence de ce goût que j'ai pour les rencontres. Mais là, c'est beaucoup plus abstrait ; il n'y a pas de visages, on ne peut pas se toucher, on ne se voit pas. Le lecteur c'est une drôle de chose. Le lecteur, c'est un peu la mort et c'est ce qui permet la vie. Mes livres sont des gestes pour continuer à vivre (Guichard, 2006 : 14-23).

Cette volonté de donner à lire le poème au lecteur, de lui offrir le geste poétique fixé sur la page, est reprise dans plusieurs poèmes de James Sacré. Par exemple, dans un poème du recueil Les mots longtemps, qu'est-ce que le poème attend? (Sacré, 2003), le poète offre à boire au lecteur, comme s'il lui offrait à partager son poème, ce que nous pourrions interpréter comme un geste à la fois de vie, d'offrande et de poésie :

Bouteille qu'on sort

De quel souvenir buisson

Braguette ou quoi d'autre

Poème pour on voudrait

Rien désir ou tendresse 
Donner à boire

T'en veux ? (Sacré, $2003: 211$ ).

Mais parfois Sacré se demande ce qui reste de son vécu quand le poème arrive au lecteur, ce que le geste mis sur la page garde de sa vie, et il finit par conclure que, même si rien de ce qu'il a vécu atteint le lecteur, il sera parvenu à réaliser le don d'un geste :

Néanmoins dans cette rencontre entre mon poème et son lecteur, je ne sais pas trop ce qui reste de mon vécu (et s'il n'en reste rien, c'est bien possible que ça soit sans importance), mais le poème n'est-il pas quand même comme une main tendue des mots qui sort d'une façon ou d'une autre de ma poche ? [...] ce que j'attends avec mon poème c'est bien qu'on réponde à son geste $[\ldots]$ ou alors dans ma solitude, ou la sienne, je pourrai simplement me prendre moi-même un peu par la main (Sacré, 2003 : 199).

Le poète sait qu'il est très probable de perdre un peu de l'essence ou de la force originelle de l'expérience vécue lorsqu'il la met sur la page. C'est-à-dire, le fait de traduire le vécu en poésie par l'intermédiaire de gestes d'écriture, représente un risque de perdre la puissance que le vécu possède en lui-même, mais le poète est prêt à prendre ce risque, parce qu'il sait en même temps, que cette transposition du vécu à la sphère poétique, lui permettra de découvrir d'autres dimensions de ce vécu, des dispositions qu'il ne pourra repérer qu'au sein du texte poétique, et qui susciteront en lui des émotions qu'il n'aurait pas découvertes autrement :

[...] même si je me désespère de perdre à travers mes mots l'intensité d'un moment vécu par exemple, je risque d'éprouver dans mon poème quelque autre plus forte émotion encore, et rien ne m'interdit de penser que ce que je crois avoir perdu l'a nourri, si même autrement qu'il me serait possible d'imaginer (Sacré, 2013b : 16).

En plus, il ne faut pas oublier que le don du texte et la réception de celui-ci par le lecteur, est une condition indispensable pour que le faire poétique puisse exister, tel que Dominique Rabaté le souligne : «Le texte n'existe que dans le présent fait à son lecteur, dans le présent réitéré de la lecture » (Rabaté, 2013 : 126).

\section{Remercier}

Finalement, tout ce processus poétique parsemé de gestes, finit avec le remerciement de l'auteur à ce qui représente la source essentielle et indispensable de sa création poétique, c'est-adire, la vie elle-même, le vécu à partir duquel James Sacré construit toute son œuvre poétique à travers des gestes poétiques. Nous pourrions dire que le processus poétique chez James Sacré réalise un parcours qui commence par un geste de vie, aussi banal soit-il, qui devient un geste d'écriture qui reste figé sur la page, et qui a la capacité d'effectuer différentes interventions poétique au sein du texte. Nous avons résumé ces possibles opérations poétiques en les associant à des actions qui permettront au sujet poétique de s'exprimer dans le poème, pour ensuite atteindre le lecteur avec un geste de don poétique, qui est finalement suivi d'un geste de remerciement au vécu, à tout ce qui a fait partie de 
l'expérience vitale du poète, sans laquelle aucun de ces gestes n'aurait jamais eu lieu : «J'aimerais bien que mes livres soient en quelque sorte des remerciements aux rencontres que j'aurais faites, soit avec des gens, soit avec des paysages. Des remerciements à ce qui m'a touché » (Guichard, 2006 : 14-23).

Mais, en faisant allusion à ce dernier geste poétique qui cherche à remercier, il faudrait également parler du remerciement du lecteur lui-même, qui est reconnaissant au geste de don poétique offert par le poète, un remerciement qu'il fait parvenir au poète à travers la lecture du texte poétique et de l'éventuelle réflexion qui en découle. D'après les mots du poète et critique Maulpoix : « La lecture est par excellence le moment où s'accomplit l'offrande. Où le texte est reçu. Le vrai remerciement du lecteur au texte donné est la pensée » (Maulpoix, 1996 : 21).

\section{Conclusion}

Gestes de vie, gestes de mots, voilà les mouvements que la poésie de James Sacré tente de reproduire à tout prix et à tout moment, parce que, comme le poète luimême avoue: "J'aime penser qu'un poème est un geste de mots » (Sacré, 2013b : 14) James Sacré ne conçoit la poésie qu'en faisant partie de cette double démarche qui réunit dans un même élan le geste et les mots ou, plutôt, les gestes par les mots, désignant même son écriture comme des « gestes-poèmes » :

Peu à peu j'ai cru comprendre que tous mes poèmes sont des gestes de mots, du sens assez précis certes, entre autres choses, mais donné dans une matière en somme corporelle (obscure) du poème. Gestes-poèmes qui font signe en même temps qu'ils disent (Sacré, 2013b : 16).

Néanmoins, James Sacré avoue que sa poésie n'est pas la seule à pouvoir produire ces gestes de mots. Il y a d'autres types de discours, loin du poétique, qui partagent cette même capacité d'accomplir des gestes poétiques, ce qui montre que le poète est conscient du manque de singularité de son faire poétique. Ce n'est pas que Sacré ne croit pas aux capacités de la poésie, mais il a une conception poétique complètement éloignée d'une vision qui représenterait l'idéalité et l'absolu, une conception plus proche de la banalité, avec un regard moins extraordinaire vis-à-vis du discours poétique. Pour James Sacré, la poésie peut indéniablement reproduire des gestes de vie à travers des gestes d'écriture, tout en reconnaissant qu'elle n'est pas la seule à posséder cette faculté :

Geste : cela qui dit, mais pas très précisément, et cependant souvent de façon intime ou forte. Le désir de sens dans le poème se défait-il pas en gestes des mots ? [...] Mais il n'y a pas que le poème qui fasse ces gestes de mots : sont-ils pas aussi bien dans les prières, dans les sournoiseries des discours politiques ou dans les plaintes du petit enfant ou du vieillard radotant? (Sacré, 2013b : 13-14).

Toute poésie, mais, particulièrement la poésie contemporaine, se caractérise par un questionnement incessant sur le faire poétique lui-même: "Mais de quoi parlons-nous, lorsque nous écrivons? Écrire, que veut ce geste ? Ce sont là 
quelques-unes des questions qui mettent, de l'intérieur, la poésie moderne en examen » (Maulpoix, $2002: 10$ ). Nous remarquons ce questionnement sur l'écriture poétique elle-même aussi chez James Sacré, qui se demande souvent pourquoi il écrit. Comme la plupart des poètes contemporains, Sacré ne trouve pas de réponse claire et parfaite à son questionnement, mais il semble être sûr que sa poésie est « un banal geste de vivant » qui cherche à se mettre en contact avec autrui ou, tout simplement, à remuer un peu dans la langue :

[...] Mais pourquoi écrire un livre de poèmes ? Pour parler avec les autres (mais on ne les voit presque jamais) ? Pour dire... mais comment croire à l'importance de ce qu'on pourrait dire ? Pour mieux dire quelque chose... mais quoi donc de si essentiel qui finit par n'être que de la poussière de mots sur des feuilles de papier blanc ? Non je ne saurais pas répondre à ce pourquoi. Sauf en imaginant qu'écrire un poème c'est juste un banal geste de vivant comme on en fait quand on travaille, quand on joue, etc., un geste de mots pour faire signe aux autres - leur faire signe de se rapprocher, ou de s'éloigner. Ou juste un geste pour soi, pour se bouger un peu dans la matière d'une langue (Sacré, 2013b : 15).

Nous pourrions conclure en affirmant que le rapport entre le vécu et le poétique est indéniable chez James Sacré, un rapport qu'il s'acharne à mettre sur la page à travers des gestes de mots, des gestes de langage, qui tentent de transmettre à la dimension poétique les gestes de vie du poète, qui sont à l'origine de toute parole poétique, de tout acte d'écriture. La poésie serait donc un espace dans lequel confluent et interagissent les mots et la vie. Néanmoins, James Sacré se montre souvent méfiant vis-à-vis de cette confluence, et il semble avoir besoin de confirmer ses gestes poétiques, en définitive, ses gestes de vie, auprès du lecteur : «La poésie ? Une affaire de langue entre le monde et les dictionnaires, une affaire de formes, entre le vécu et la langue ; si c'est geste de vivant ou rien, peut-être qu'un lecteur me dira » (Sacré, 2013b : 15).

Mais James Sacré va plus loin encore en déclarant que tout son travail poétique, depuis le premier recueil qu'il a publié en 1965, répond à un même souci, à une même obsession pourrions-nous dire, et c'est la préoccupation pour la possibilité de créer un espace - celui de la dimension poétique - où pourraient converger et se réunir l'écriture et la vie. Tout le travail poétique de James Sacré sera donc fortement marqué dès le début par cette inquiétude, et il semble avoir trouvé une réponse possible à ce souci de convergence entre écriture et vie, grâce à la présence des gestes poétiques dans son écriture : des gestes de mots qui imitent les gestes de vie, parvenant ainsi à créer une écriture que nous pourrions qualifier comme étant une écriture de gestes de vie :

Tout le livre [Un paradis de poussières] comme tous mes livres depuis Relation (mon premier en 1965), est probablement un même trouble écrit (le poème comme un précipité du corps avec le langage) où s'épuise et persiste (s'émerveille, ou s'impatiente et se désespère) une interrogation sur la possibilité d'une rencontre entre les mots et le monde, l'écriture du poème et sa lecture, l'autre et le même, le privé et le public (Sacré, 2013b : 17). 


\section{REFERENCES BIBLIOGRAPHIQUES}

Bancquart, M.-C., (2010) Explorer l'incertain. Coaraze, L'amourier.

Emaz, A., (2009) Cambouis. Corlet à Condé-sur-Noireau, Éditions du Seuil.

Emaz, A., (2012) Cuisine. Paris, Publie-papier.

Guichard, T., (juillet-août 2006) « James Sacré, la poésie au cœur », in Le Matricule des Anges. No 75, pp. 14-23.

Laplace, A., (mars 1984) «James Sacré, Rencontre avec Pierre Ceysson, Jacques Imbert, Dominique Celette, André Reiss, Luc Pavillon, Frédéric Chambe, Alain Frémy,Geneviève Vidal, Jacqueline Merville, Marc Porcu », in Cahier de Poésie-Rencontres. $\mathrm{N}^{\circ} 11,8$.

Lewers, D., (2010) Introduction à la poésie moderne et contemporaine. Paris, Armand Colin.

Maulpoix, J.-M., (1995) La poésie malgré tout. Paris, Mercure de France.

Maulpoix, J.-M., (1998) Domaine public. Paris, Mercure de France.

Maulpoix, J.-M., (2002) Le poète perplexe. Paris, José Corti.

Maulpoix, J.-M., (2005) Adieux au poème. Paris, José Corti.

Maxence, J.-L., (2014) Au tournant du siècle: Regard critique sur la poésie française contemporaine. Saint-Amand-Montrond, Seghers.

Pinson, J.-C., (2002) Sentimentale et naïve: Nouveaux essais sur la poésie contemporaine. Sayssel, Champ Vallon.

Rabaté, D., (2013) Gestes lyriques. Mayenne, Éditions Corti.

Sacré, J., (2000) Si peu de terre, tout. Bassac, Le dé bleu.

Sacré, J. (2003) Les mots longtemps, qu'est-ce que le poème attend? Saint-Benoîtdu-Sault, Éditions Tarabuste.

Sacré, J., (2010a) Tissus mis par terre et dans le vent. Bègles, Le Castor Astral.

Sacré, J., (2010b) America solitudes. Marseille, André Dimanche Éditeur.

Sacré, J., (2012a) Si les felos traversent par nos poèmes? Remoulins-sur-Gardon, Éditions Jacques Brémond.

Sacré, J., (2012b) Affaires d'écriture (Ancrits divers). Saint-Benoît-du-Sault, Éditions Tarabuste.

Sacré, J., (2013a) Donne-moi ton enfance. Saint-Benoît-du-Sault, Éditions Tarabuste.

Sacré, J., (2013b) Parler avec le poème. Genève, Éditions la Baconnière.

\section{SITOGRAPHIE}

Maulpoix, J.-M., (décembre 2012) Conférence à l'Université Toulouse le Mirail: Extraire la beauté $d u$ trivial. [En ligne]. Disponible sur: https://www.canalu.tv/video/universite_toulouse_ii_le_mirail/extraire_la_beaute _du_trivial_jean_michel_maulpoix.11668 [Dernier accès le 15 mars 2014].

Rimbaud, A., (1871) Extrait de la lettre à Paul Demeny dite lettre du voyant, 15 mai 1871. [En ligne]. Disponible sur : https://fr.wikisource.org/wiki/Lettre_de_Rimbaud_\%C3\%A0_Paul_Demeny__15_mai_1871 [Dernier accès le 13/02/2014.] 